\title{
CONVERGENCE OF NONLINEAR SEMIGROUPS AND APPROXIMATION SCHEMES TO WELL- POSED CAUCHY PROBLEM IN BANACH SPACES
}

\author{
By \\ Ki Sik HA \\ (Received Apr. 30, 1971)
}

\section{§1. Introduction.}

Throughout this paper $X$ is a Banach space. Let $X_{0}$ be a subset of $X$, and let $\{T(t) ; t \geqslant 0\}$ be a family of one-parameter nonlinear operators from $X_{0}$ into itself satisfying the following conditions:

(i) $T(0)=I$ (the identity), $T(t+s)=T(t) T(s)$ for $t, s \geqslant 0$,

(ii) $\|T(t) x-T(t) y\| \leqslant\|x-y\|$ for $t \geqslant 0$ and $x, y \in X_{0}$,

(iii) $\lim _{t \rightarrow 0+} T(t) x=x$ for $x \in X_{0}$.

The family $\{T(t) ; t \geqslant 0\}$ is called a contraction semigroup on $X_{0}$.

We shall deal with multi-valued operators. Let $A$ be a multi-valued nonlinear operator in $X$. By this we mean that $A$ assigns to each $x \in X$ a subset $A x$ of $X . \quad A x$ may be empty for some $x \in X$. By definition the domain $D(A)$ of $A$ is the set of all $x \in X$ for which $A x$ is not empty. We define the range $R(A)$ of $A$ as the union of the $A x$ for all $x \in D(A)$. We define for $x \in D(A)$

$$
\|A x\|=\inf \left\{\left\|x^{\prime}\right\| ; x^{\prime} \in A x\right\} .
$$

Definition 1.1. A multi-valued operator $A$ in $X$ is said to be dissipative if for each $\alpha>0, x, y \in D(A), x^{\prime} \in A x$ and $y^{\prime} \in A y$

$$
\left\|x-y-\alpha\left(x^{\prime}-y^{\prime}\right)\right\| \geqslant\|x-y\| \text {. }
$$

Let $A$ be dissipative in $X$. Then for $\alpha>0$ we can define single-valued operators

$$
J_{\alpha}=(I-\alpha A)^{-1}: R(I-\alpha A)\left(=D\left(J_{\alpha}\right)\right) \rightarrow D(A)
$$


such that $\left\|J_{\alpha} x-J_{\alpha} y\right\| \leqslant\|x-y\|$ for $x, y \in D\left(J_{\alpha}\right)$. We set $A_{\alpha}=\alpha^{-1}\left(J_{\alpha}\right.$ $-I)$. The implication

$$
A_{\alpha} x \in A J_{\alpha} x \text { for } x \in D\left(J_{\alpha}\right)
$$

is well-known. Recently M.G. Crandall and T.M. Liggett [3] proved the following lemma and theorem.

Lemma 1.2. Let $A$ be dissipative in $X$. Then

(a) for $\alpha, \beta>0$ and $x \in D\left(J_{\alpha}\right)$,

$$
\begin{aligned}
& \frac{\beta}{\alpha} x+\frac{\alpha-\beta}{\alpha} J_{\alpha} x \in D\left(J_{\beta}\right), \text { and } \\
& J_{\alpha} x=J_{\beta}\left(\frac{\beta}{\alpha} x+\frac{\alpha-\beta}{\alpha} J_{\alpha} x\right),
\end{aligned}
$$

(b) for $\alpha \geqslant \beta>0, n \geqslant m>0$ and $x \in D\left(J_{\alpha}^{m}\right) \cap D\left(J_{\alpha}^{n}\right) \cap D(A)$

$$
\begin{aligned}
& \left\|J_{\beta}^{n} x-J_{\alpha}^{m} x\right\| \leqslant\left(\left((n \beta-m \alpha)^{2}+n \beta(\alpha-\beta)\right)^{1 / 2}\right. \\
& \left.+\left(m \alpha(\alpha-\beta)+(m \alpha-n \beta)^{2}\right)^{1 / 2}\right)\|A x\|,
\end{aligned}
$$

where $m, n$ are integers.

Theorem 1.3. Let $A$ be dissipative in $X$ satisfying

$$
R(I-\alpha A) \supset \overline{D(A)} \text { for } \alpha>0 .
$$

Then there exists a contraction semigroup $\{T(t) ; t \geqslant 0\}$ on $\overline{D(A)}$ such that for each $x \in \overline{D(A)}$

$$
T(t) x=\lim _{\alpha \rightarrow 0+} J_{\alpha}^{[t / \alpha]} x
$$

uniformly for $t$ in every bounded interval of $[0, \infty)$, and

$$
\|T(t) x-T(s) x\| \leqslant|t-s|\|A x\|
$$

for $t, s \geqslant 0$ and $x \in D(A)$ where [] denotes the Gaussian bracket.

The purpose of this paper is to extend the Kato-Trotter Theorem in $\mathrm{K}$. Yosida [9] to the case of nonlinear contraction semigroups given in Theorem 1.3, and to consider the convergence of the approximation scheme 


$$
\left\{\begin{array}{l}
\frac{d}{d t} u_{n}(t) \in A_{n} u(t) \\
u_{n}(0)=x
\end{array}\right.
$$

in $X$ to the Cauchy problem

$$
\left\{\begin{array}{l}
\frac{d}{d t} u(t) \in A u(t) \\
u(0)=x
\end{array}\right.
$$

in $X$, well-posed in the sense of semigroup when $A_{n}$ approximates $A$ in the sense of (3.3), where $A$ and $A_{n}$ are nonlinear multi-valued operators.

Let $A$ be dissipative in $X$ satisfying (1.3). Setting $n=[t / \beta]$ and $m=[t / \alpha]$ in (1.2), we have for $x \in D(A)$

$$
\begin{aligned}
\| J_{\beta}^{[t / \beta]} x & -J_{\alpha}^{[t / \alpha]} x \| \\
& \leqslant\left(\left(\alpha^{2}+t(\alpha-\beta)\right)^{1 / 2}+\left(t(\alpha-\beta)+\beta^{2}\right)^{1 / 2}\right)\|A x\| .
\end{aligned}
$$

As $\beta \rightarrow 0+$, it follows from Theorem 1.3 that

$$
\left\|T(t) x-J_{\alpha}^{[t / \alpha]} x\right\| \leqslant\left(\left(\alpha^{2}+t \alpha\right)^{1 / 2}+(t \alpha)^{1 / 2}\right)\|A x\|
$$

for $x \in D(A)$.

$\S 2$ concerns the convergence of contraction semigroups given in Theorem 1.3.

In $\S 3$ we deal with the convergence of the solutions of approximation schemes to Cauchy problem well-posed in the sense of a contraction semigroup.

\section{$\S 2 . \quad$ Convergence of contraction semigroups.}

Let $\left\{A_{n}\right\}$ be a sequence of dissipative operators in $X$ satisfying

$$
R\left(I-\alpha A_{n}\right) \supset \overline{\operatorname{co} D\left(A_{n}\right)} \text { for } \alpha>0,
$$

and put $J_{\alpha, n}=\left(I-\alpha A_{n}\right)^{-1}$ for $\alpha>0$.

Theorem 2.1. Let $\left\{A_{n}\right\}$ be a sequence of dissipative operators in $X$ satisfying (2.1). If 


$$
D \subset D\left(A_{n}\right) \text { for every } n
$$

and

$$
\lim _{n \rightarrow \infty} J_{\alpha_{0}, n} x(x \in \overline{\operatorname{co} D}) \text { exists in } D \text { for some } \alpha_{0}>0 \text {, }
$$

then, denoting by $J_{\alpha_{0}} x$ the limit value of (2.3), there exists a dissipative operator $A$ in $X$ such that $J_{\alpha_{0}}=\left(I-\alpha_{0} A\right)^{-1}$ for $\alpha_{0}>0$. In addition if (1.3) is satisfied then $\lim _{n \rightarrow \infty} T_{n}(t) x=T(t) x$ for $x \in \overline{D(A)}$ uniformly for $t$ in every bounded interval of $[0, \infty)$, where $\left\{T_{n}(t) ; t \geqslant 0\right\},\{T(t) ; t \geqslant 0\}$ are contraction semigroups given in Theorem 1.3 by $A_{n}$ and $A$ respectively and co $D$ denotes the convex hull of $D$.

The following two lemmas make complete the proof of Theorem 2.1.

Lemma 2.2. Let $\left\{A_{n}\right\}$ be a sequence of dissipative operators in $X$ satisfying (2.1). If the conditions (2.2) and (2.3) are satisfied then

(a) $\lim _{n \rightarrow \infty} J_{\alpha, n} x, x \in \overline{\operatorname{co} D}$ exists in $D$ for all $\alpha>0$.

We denote by $J_{\alpha} x$ the limit value of (a).

(b) There exists a dissipative operator $A$ in $X$ such that $J_{\alpha}=(I-$ $\alpha A)^{-1}$ for all $\alpha>0$.

Proof. (a) Since $J_{\alpha_{0}, n}$ is contraction, so is $J_{\alpha_{0}}$. We first prove that for $\alpha>0, \frac{\alpha}{\alpha_{0}} I+\left(1-\frac{\alpha}{\alpha_{0}}\right) J_{\alpha_{0}}$ maps $\overline{\operatorname{co} D}$ onto itself. If $x \in \overline{\operatorname{co} D}$, then $J_{\alpha_{0}} x$ $\in \overline{\operatorname{co} D}$, and hence $\frac{\alpha}{\alpha_{0}} x+\left(1-\frac{\alpha}{\alpha_{0}}\right) J_{\alpha_{0}} x \in \overline{\operatorname{co} D}$. Then taking $y \in \overline{\operatorname{co} D}$, $\frac{\alpha_{0}}{\alpha} y+\left(1-\frac{\alpha_{0}}{\alpha}\right) J_{\alpha_{0}} x \in \overline{\operatorname{co} D}$. We define an operator $K$ by $K x=\frac{\alpha_{0}}{\alpha} y+$ $\left(1-\frac{\alpha_{0}}{\alpha}\right) J_{\alpha_{0}} x$ for $x \in \overline{\operatorname{co} D}$. If $\alpha>\frac{\alpha_{0}}{2}, K$ is a strict contraction operator from $\overline{\operatorname{co} D}$ into itself. In fact for $x_{1}, x_{2} \in \overline{\operatorname{co} D}$,

$$
\begin{aligned}
& \left\|K x_{1}-K x_{2}\right\| \\
& =\left\|\frac{\alpha_{0}}{\alpha} y+\left(1-\frac{\alpha_{0}}{\alpha}\right) J_{\alpha_{0}} x_{1}-\left(\frac{\alpha_{0}}{\alpha} y+\left(1-\frac{\alpha_{0}}{\alpha}\right) J_{\alpha_{0}} x_{2}\right)\right\| \\
& =\left|1-\frac{\alpha_{0}}{\alpha}\right|\left\|J_{\alpha_{0}} x_{1}-J_{\alpha_{0}} x_{2}\right\|
\end{aligned}
$$




$$
\leqslant\left|1-\frac{\alpha_{0}}{\alpha}\right|\left\|x_{1}-x_{2}\right\|
$$

By the fixed point theorem there exists a unique element $x \in \overline{\operatorname{co} D}$ such that $K x=x$. Thus we have $x=\frac{\alpha_{0}}{\alpha} y+\left(1-\frac{\alpha_{0}}{\alpha}\right) J_{\alpha_{0}} x$. From this we obtain $y=\frac{\alpha}{\alpha_{0}} x+\left(1-\frac{\alpha}{\alpha_{0}}\right) J_{\alpha_{0}} x$, and

$$
\overline{\operatorname{co} D}=\left\{y ; y=\frac{\alpha}{\alpha_{0}} x+\left(1-\frac{\alpha}{\alpha_{0}}\right) J_{\alpha_{0}} x, x \in \overline{\operatorname{co} D}\right\}
$$

for $\alpha>\frac{\alpha_{0}}{2}$. Next we show that $\lim _{n \rightarrow \infty} J_{\alpha, n} x, x \in \overline{\operatorname{co} D}$ exists in $D$ for all $\alpha>0$. For $x, y \in \overline{\operatorname{co} D}$ and $\alpha>\frac{\alpha_{0}}{2}$, put $y=\frac{\alpha}{\alpha_{0}} x+\left(1-\frac{\alpha}{\alpha_{0}}\right) x$. Then we obtain

$$
\begin{aligned}
\| J_{\alpha_{0}} x-J_{\alpha, n} y & \leqslant\left\|J_{\alpha_{0}} x-J_{\alpha_{0}, n} x\right\|+\left\|J_{\alpha_{0}, n} x-J_{\alpha, n} y\right\| \\
& \leqslant\left\|J_{\alpha_{0}} x-J_{\alpha_{0}, n} x\right\| \\
+ & \left\|J_{\alpha, n}\left(\frac{\alpha}{\alpha_{0}} x+\left(1-\frac{\alpha}{\alpha_{0}}\right) J_{\alpha_{0}, n} x\right)-J_{\alpha, n}\left(\frac{\alpha}{\alpha_{0}} x+\left(1-\frac{\alpha}{\alpha_{0}}\right) J_{\alpha_{0}} x\right)\right\| \\
& \leqslant\left\|J_{\alpha_{0}} x-J_{\alpha_{0}, n} x\right\|+\left|1-\frac{\alpha}{\alpha_{0}}\right|\left\|J_{\alpha_{0}} x-J_{\alpha_{0}, n} x\right\| \\
& =\left(1+\left|1-\frac{\alpha}{\alpha_{0}}\right|\right)\left\|J_{\alpha_{0}} x-J_{\alpha_{0}, n} x\right\| \rightarrow 0
\end{aligned}
$$

as $n \rightarrow \infty$. Here we have used Lemma 1.2(a). Therefore $\lim _{n \rightarrow \infty} J_{\alpha, n} y, y$ $\in \overline{\operatorname{co} D}$ exists for $\alpha>\frac{\alpha_{0}}{2}$. Since $\alpha_{1}=\frac{2}{3} \alpha_{0}>\frac{\alpha_{0}}{2}, \lim _{n \rightarrow \infty} J_{\alpha_{1}, n} y, y \in \overline{\operatorname{co} D}$ exists. Then in an analogous consideration we find that $\lim _{n \rightarrow \infty} J_{\alpha, n} y, y \in \overline{\operatorname{co} D}$ exists for all $\alpha>\frac{\alpha_{0}}{3}$. Repeating this process, we have that $\lim _{n \rightarrow \infty} J_{\alpha, n} x, x \in \overline{\operatorname{co} D}$ for all $\alpha>0$. By Lemma 1.2(a), $J_{\alpha, n} x=J_{\alpha_{0}, n}\left(\frac{\alpha_{0}}{\alpha} x+\left(1-\frac{\alpha_{0}}{\alpha}\right) J_{\alpha, n} x\right)$. Since $J_{\alpha, n}$ and $J_{\alpha}$ are contraction, we obtain 


$$
J_{\alpha} x=J_{\alpha_{0}}\left(\frac{\alpha_{0}}{\alpha} x+\left(1-\frac{\alpha_{0}}{\alpha}\right) J_{\alpha} x\right)
$$

for $x \in \overline{\operatorname{co} D}$. This equality means that $R\left(J_{\alpha}\right)=R\left(J_{\alpha_{0}}\right)$, and hence by (2.3) $\lim _{n \rightarrow \infty} J_{\alpha, n} x=J_{\alpha} x \in D, x \in \overline{\operatorname{co} D}$ for all $\alpha>0$.

(b) Since $R\left(J_{\alpha}\right)$ is independent of $\alpha>0$, we define $J_{\alpha}^{-1}$ by $J_{\alpha}^{-1} x=\{y$ $\left.\in \overline{\operatorname{co} D} ; J_{\alpha} y=x\right\}$ for $x \in R\left(J_{\alpha}\right)$. Then $\frac{1}{\alpha_{0}}\left(x-J_{\alpha_{0}}^{-1} x\right)=\frac{1}{\alpha}\left(x-J_{\alpha}^{-1} x\right)$. Indeed, by (2.4) for $y \in \overline{\operatorname{co} D}$ we have $\left(\frac{\alpha_{0}}{\alpha} y+\left(1-\frac{\alpha_{0}}{\alpha}\right) J_{\alpha} y\right) \in J_{\alpha_{0}}^{-1} J_{\alpha} y$, and hence $\frac{1}{\alpha}\left(J_{\alpha} y-y\right) \in \frac{1}{\alpha_{0}}\left(J_{\alpha} y-J_{\alpha_{0}}^{-1} J_{\alpha} y\right)$. There exists $x \in R\left(J_{\alpha}\right)$ such that $x=J_{\alpha} y$. Thus we obtain $\frac{1}{\alpha}\left(x-J_{\alpha}^{-1} x\right)\left(\frac{1}{\alpha_{0}}\left(x-J_{\alpha_{0}}^{-1} x\right)\right.$. Set $D(A)=R\left(J_{\alpha}\right)$ for $\alpha>0$ and we define $A$ by $A x=\frac{1}{\alpha}\left(x-J_{\alpha}^{-1} x\right)$ for $x \in D(A)$ Then $J_{\alpha}=$ $(I-\alpha A)^{-1}$ for all $\alpha>0$. We shall show that $A$ is dissipative. In fact, for $x, y \in D(A)$ and $x^{\prime} \in A x, y^{\prime} \in A y$ we have $J_{\alpha}\left(x-\alpha x^{\prime}\right)=x$ and $J_{\alpha}(y$ $\left.-\alpha y^{\prime}\right)=y$ for $\alpha>0$. Since $J_{\alpha}$ is contraction we obtain

$$
\begin{aligned}
\|x-y\| & =\left\|J_{\alpha}\left(x-\alpha x^{\prime}\right)-J_{\alpha}\left(y-y^{\prime}\right)\right\| \\
& \leqslant\left\|x-y-\alpha\left(x^{\prime}-y^{\prime}\right)\right\|,
\end{aligned}
$$

whcih means that $A$ is dissipative in $X$.

Lemma 2.3. Let $\left\{A_{n}\right\}$ be a sequence of dissipative operators in $X$ satisfying (2.1). If the conditions (2.2), (2.3) in Theorem 2.1 and (1.3) are satisfied and if $\left\{T_{n}(t) ; t \geqslant 0\right\},\{T(t) ; t \geqslant 0\}$ are contraction semigroups given in Theorem 1.3 by $A_{n}$ and $A$ respectively, then $\lim _{n \rightarrow \infty} T_{n}(t) x=T(t) x$ for $x \in \overline{D(A)}$ uniformly for $t$ in every bounded interval of $[0, \infty)$. Where $A$ is the dissipative operator given in Lemma 2.2 .

Proof. Put $A_{\alpha, n}=\alpha^{-1}\left(J_{\alpha, n}-I\right)$ and $A_{\alpha}=\alpha^{-1}\left(J_{\alpha}-I\right)$ for $\alpha>0$. Since $\lim _{n \rightarrow \infty} J_{\alpha, n} x=J_{\alpha} x, x \in \overline{\operatorname{co} D}$ for all $\alpha>0$, we have $\lim _{n \rightarrow \infty} A_{\alpha, n} x=A_{\alpha} x, x \in \overline{\operatorname{co} D}$ for all $\alpha>0$. Therefore there exists $M>0$ such that $\left\|A_{\alpha, n} x\right\| \leqslant M$ and $\left\|A_{\alpha} x\right\|$ $\leqslant M$. By the definition of $A$, it follows that $D(A) \subset D \subset D\left(A_{n}\right)$. We fix $y \in \overline{D(A)}$ and $\alpha_{0}>0$. Then

$$
\left\|T_{n}(t) J_{\alpha_{0}} y-T(t) J_{\alpha_{0}} y\right\|
$$




$$
\begin{aligned}
& \leqslant\left\|T_{n}(t) J_{\alpha_{0}} y-J_{\alpha, n}^{[t / \alpha]} J_{\alpha_{0}} y\right\| \\
& +\left\|J_{\alpha, n}^{[t / \alpha]} J_{\alpha_{0}} y-J_{\alpha}^{[t / \alpha]} J_{\alpha_{0}} y\right\| \\
& +\left\|J_{\alpha}^{[t / \alpha]} J_{\alpha_{0}} y-T(t) J_{\alpha_{0}} y\right\| .
\end{aligned}
$$

We estimate the first term of the left side in the above inequality as follows.

$$
\begin{aligned}
\left\|T_{n}(t) J_{\alpha_{0}} y-J_{\alpha, n}^{[t / \alpha]} J_{\alpha_{0}} y\right\| \\
\quad \leqslant\left\|T_{n}(t) J_{\alpha_{0}} y-T_{n}(t) J_{\alpha_{0}, n} y\right\| \\
\quad+\left\|T_{n}(t) J_{\alpha_{0}, n} y-J_{\alpha, n}^{[t / \alpha]} J_{\alpha_{0}, n} y\right\| \\
\quad+\left\|J_{\alpha, n}^{[t \mid \alpha]} J_{\alpha_{0}, n} y-J_{\alpha, n}^{[t / \alpha]} J_{\alpha_{0}} y\right\| \\
\quad \leqslant 2\left\|J_{\alpha_{0}} y-J_{\alpha_{0}, n} y\right\|+\left\|T_{n}(t) J_{\alpha_{0}, n} y-J_{\alpha, n}^{[t / \alpha]} J_{\alpha_{0}, n} y\right\| \\
\quad \leqslant 2\left\|J_{\alpha_{0}} y-J_{\alpha_{0}, n} y\right\|+\left(\left(\alpha^{2}+t \alpha\right)^{1 / 2}+(t \alpha)^{1 / 2}\right)\left\|A_{n} J_{\alpha_{0}, n} y\right\| .
\end{aligned}
$$

Since $\left\|A_{n} J_{\alpha_{0}, n} y\right\| \leqslant\left\|A_{\alpha_{0}, n} y\right\| \leqslant M$, we have

$$
\begin{aligned}
& \left\|T_{n}(t) J_{\alpha_{0}} y-J_{\alpha, n}^{[t, \alpha]} J_{\alpha_{0}} y\right\| \\
& \quad \leqslant 2\left\|J_{\alpha_{0}} y-J_{\alpha_{0}, n} y\right\|+\left(\left(\alpha^{2}+t \alpha\right)^{1 / 2}+(t \alpha)^{1 / 2}\right) M .
\end{aligned}
$$

Also

$$
\begin{aligned}
&\left\|J_{\alpha}^{[t / \alpha]} J_{\alpha_{0}} y-T(t) J_{\alpha_{0}} y\right\| \\
& \leqslant\left(\left(\alpha^{2}+t \alpha\right)^{1 / 2}+(t \alpha)^{1 / 2}\right)\left\|A J_{\alpha_{0}} y\right\| \\
& \leqslant\left(\left(\alpha^{2}+t \alpha\right)^{1 / 2}+(t \alpha)^{1 / 2}\right)\left\|A_{\alpha_{0}} y\right\| \\
& \leqslant\left(\left(\alpha^{2}+t \alpha\right)^{1 / 2}+(t \alpha)^{1 / 2}\right) M .
\end{aligned}
$$

Here we have used (1.1) and (1.4). Thus

$$
\begin{aligned}
\| T_{n}(t) J_{\alpha_{0}} y & -T(t) J_{\alpha_{0}} y \| \\
& \leqslant 2\left\|J_{\alpha_{0}} y-J_{\alpha_{0}, n}\right\| \\
& +\left\|J_{\alpha, n}^{[t / \alpha]} J_{\alpha_{0}} y-J^{[t / \alpha]} J_{\alpha_{0}} y\right\| \\
& +2\left(\left(\alpha^{2}+t \alpha\right)^{1 / 2}+(t \alpha)^{1 / 2}\right) M .
\end{aligned}
$$


Let $t_{0} \geqslant 0$ and choose $\alpha_{1}>0$ such that for each $\varepsilon>0$,

$$
2\left(\left(\alpha_{1}^{2}+t \alpha_{1}\right)^{1 / 2}+\left(t \alpha_{1}\right)^{1 / 2}\right) M<\varepsilon, 0 \leqslant t \leqslant t_{0} .
$$

Then

$$
\begin{aligned}
\| T_{n}(t) & J_{\alpha_{0}} y-T(t) J_{\alpha_{0}} y \| \\
& \leqslant 2\left\|J_{\alpha_{0}} y-J_{\alpha_{0}, n} y\right\| \\
& +\left\|J_{\alpha_{1}, n}^{[t / \alpha]} J_{\alpha_{0}} y-J_{\alpha_{1}}^{[t / \alpha]} J_{\alpha_{0}} y\right\| \\
& +2\left(\left(\alpha_{1}^{2}+t \alpha_{1}\right)^{1 / 2}+\left(t \alpha_{1}\right)^{1 / 2}\right) M .
\end{aligned}
$$

An $n \rightarrow \infty,\left\|T_{n}(t) J_{\alpha_{0}} y-T(t) J_{\alpha_{0}} y\right\| \rightarrow 0,0 \leqslant t \leqslant t_{0}$. Since $R\left(J_{\alpha_{0}}\right)=D(A)$, there exists $x \in D(A)$ such that $x=J_{\alpha_{0}} y$. Therefore it follows that

$$
\lim _{n \rightarrow \infty} T_{n}(t) x=T(t) x \quad \text { for } x \in D(A)
$$

uniformly for $t$ in every bounded interval of $[0, \infty)$. Since $T_{n}(t), T(t)$ are contraction, (2.5) holds true for $x \in \overline{D(A)}$.

The proof of Theorem 2.1 is complete.

Remark 2.4. Recently H. Brezis and A. Pazy [2] proved Theorem 2.1 in the case that $\left\{A_{n}\right\}$ is a sequence of maximal dissipative operators satisfying $R\left(I-\alpha A_{n}\right) \supset \overline{\operatorname{co} D\left(A_{n}\right)}$ in a Hilbert space $\mathrm{H}$, and I. Miyadera [5] proved the Theorem in the case that $\left\{A_{n}\right\}$ is a sequence of m-dissipative operators in $X$ whose dual $X^{*}$ is uniformly convex.

\section{§3. Convergence of approximation schemes.}

We consider the Cauchy problem

$$
\left\{\begin{array}{l}
\frac{d}{d t} u(t) \in A u(t) \\
u(0)=x
\end{array}\right.
$$

in $X$, where $A$ is a given multi-valued operator in $X$. A single-valued mapping $u(t):[0, \infty) \rightarrow X$ is called a solution of the Cauchy problem if for $t \geqslant 0 u(t)$ is Lipschitz continuous and for a.e. $t \geqslant 0$ it strongly differentiable belongs to $D(A)$ and satisfies (3.1). If $A$ is a closed operator 
and

$$
R(I-\alpha A) \supset D(A) \quad \text { for } \alpha>0,
$$

then (1.3) holds true because $R(I-\alpha A)$ is closed.

The following theorems are due to H. Brezis and A. Pazy [1] and I. Miyadara [6] respectively.

Theorem 3.1. Let $A$ be a dissipative operator in $X$ satisfying (3.2) and let $x \in D(A)$. If $u(t)$ is a solution of the Cauchy problem, then $u(t)$ $=\lim _{\alpha \rightarrow 0+} J_{\alpha}^{[t \mid \alpha]} x$ uniformly for $t$ in every bounded interval of $[0, \infty)$.

Theorem 3.2. Let $A$ be a closed dissipative operator in $X$ satisfying (3.2) and let $\{T(t) ; t \geqslant 0\}$ be a contraction semigroup on $\overline{D(A)}$ given in Theorem 1.3 by $A$. If $T(t) x$ for $x \in D(A)$ is strongly differentiable at a.e. $t \geqslant 0$ then it is a unique solution of the Cauchy problem.

Let $\left\{A_{n}\right\}$ be a sequence of multi-valued operators in $X$ satisfying $D(A) \subset D\left(A_{n}\right)$ which approximates $A$ in the sence that

$$
\lim _{n \rightarrow \infty} A_{n} x=A x \quad \text { for } x \in D(A),
$$

i.e. for each $x^{\prime} \in A x$ there is a sequence $\left\{x_{n}^{\prime}\right\}, x_{n}^{\prime} \in A_{n} x$, such that $x_{n}^{\prime} \rightarrow x^{\prime}$. Then we obtain the sequence of equations

$$
\left\{\begin{array}{l}
\frac{d}{d t} u_{n}(t) \in A_{n} u_{n}(t) \\
u_{n}(0)=x
\end{array}\right.
$$

in $X$, which is called an approximation scheme to the Cauchy problem.

Let $\left\{A_{n}\right\}$ be a sequence of dissipative operators in $X$ satisfying

$$
R\left(I-\alpha A_{n}\right) \supset D\left(A_{n}\right) \quad \text { for } \alpha>0 .
$$

By Theorem 3.1 the solution $u_{n}(t)$ of (3.4) for each $n$ is represented as

$$
u_{n}(t) \equiv T_{n}(t) x=\lim _{\alpha \rightarrow 0+} J_{\alpha, n}^{[t / \alpha]} x \quad \text { for } x \in D\left(A_{n}\right)
$$

uniformly for $t$ in every bounded interval of $[0, \infty)$.

Definition 3.3. The Cauchy problem is said to be well-posed in the 
sense of semigroup if there exists a contraction semigroup on $\overline{D(A)}$ such that, for $x \in D_{1} \subset \overline{D(A)}, u(t)=T(t) x$ is a unique solution of the Cauchy problem.

We shall consider the problem which the solution $u_{n}(t)$ of (3.4) converges to the solution $u(t)$ of the Cauchy problem well-posed in the sense of semigroup.

Theorem 3.4. Let $\left\{A_{n}\right\}$ be a sequence of dissipative operators in $X$ satisfying (2.1) and let $u_{n}(t)=T_{n}(t) x, x \in D\left(A_{n}\right)$ be a solution of (3.4). Let $A$ be a closed dissipative operator in $X$ satisfying (3.2). If $D(A)$ $C D\left(A_{n}\right)$ for each $n$ and $A_{n} x \rightarrow A x$ for $x \in D(A)$ then the Cauchy problem is well-posed in the sense of semigroup and we have

$$
\lim _{n \rightarrow \infty} T_{n}(t) x=T(t) x \quad \text { for } x \in D(A)
$$

uniformly for $t$ in every bounded interval of $[0, \infty)$, where $\{T(t) ; t \geqslant 0\}$ is a contraction semigroup on $\overline{D(A)}$ given in Theorem 1.3 and $u(t)=T(t) x$, $x \in D(A)$ is strongly differentiable at a.e. $t \geqslant 0$.

Proof. We note that $T(t) x=\lim _{\alpha \rightarrow 0+} J_{\alpha}^{[t / \alpha]} x$ for $x \in \overline{D(A)}$ uniformly for $t$ in every bounded interval of $[0, \infty)$. By Theorem 3.2, $T(t) x$ for $x$ $\in D(A)$ is a unique solution of the Cauchy problem. Thus the Cauchy problem is well-posed in the sense of semigroup. Let $x \in D(A)$. There exists $y \in D(A)$ such that for each $y^{\prime} \in A y$, there is a sequence $\left\{y_{n}^{\prime}\right\}$, $y_{n}^{\prime} \in A_{n} y, y_{n}^{\prime} \rightarrow y^{\prime}$ and $x=y-\alpha y^{\prime}$ for $\alpha>0$. We have

$$
\begin{aligned}
\| J_{\alpha, n} x- & J_{\alpha} x\|=\| J_{\alpha, n} x-y \| \\
& =\left\|J_{\alpha, n} x-J_{\alpha, n}\left(y-\alpha y_{n}^{\prime}\right)\right\| \\
& \leqslant\left\|x-y+\alpha y_{n}^{\prime}\right\| \\
& =\alpha\left\|y_{n}^{\prime}-y^{\prime}\right\| \rightarrow 0
\end{aligned}
$$

for some $\alpha>0$ as $n \rightarrow \infty$. It follows that $\lim _{n \rightarrow \infty} J_{\alpha, n} x=J_{\alpha} x, x \in D(A)$ for all $\alpha>0$. Thus, using (3.6) and an analogy of the proof of Lemma 2.3, we have (3.7).

TheOREM 3.5. Let $\left\{A_{n}\right\}$ be a sequence of dissipative operators in $X$ 
satisfying (2.1) and let $u_{n}(t)=T_{n}(t) x, x \in D\left(A_{n}\right)$ be a solution of (3.4). Suppose that

$$
\begin{gathered}
\bar{D} \subset D\left(A_{n}\right) \text { for each } n, \\
\lim _{n \rightarrow \infty} A_{n} x \quad(=A x) \text { exists for } x \in D,
\end{gathered}
$$

and

$$
R(I-\alpha A) \supset \overline{\operatorname{co} D(A)} \text { for } \alpha>0 .
$$

Then the Cauchy problem

$$
\left\{\begin{array}{l}
\frac{d}{d t} u(t) \in \bar{A} u(t) \\
u(0)=x
\end{array}\right.
$$

in $X$, is well-posed in the sense of semigroup, and

$$
\lim _{n \rightarrow \infty} T_{n}(t) x=T(t) x \text { for } x \in D(A)
$$

uniformly for $t$ in every bounded interval of $[0, \infty)$, where $\{T(t) ; t \geqslant 0\}$ is a contraction semigroup on $\overline{D(\bar{A})}$ and $T(t) x, x \in \overline{D(\bar{A})}$ is strongly differentiable a.e. $t \geqslant 0$.

Proof. It follows from (3.9) that for $x, y \in D(A)$ and $x^{\prime} \in A x, y^{\prime}$ $\in A y$, there exists $\left\{x_{n}^{\prime}\right\},\left\{y_{n}^{\prime}\right\}, x_{n}^{\prime} \in A_{n} x, y_{n}^{\prime} \in A_{n} y$ such that $x_{n}^{\prime} \rightarrow x^{\prime}, y_{n}^{\prime} \rightarrow y^{\prime}$ as $n \rightarrow \infty$. By the dissipativity of $A_{n}$,

$$
\left\|x-y-\alpha\left(x_{n}^{\prime}-y_{n}^{\prime}\right)\right\| \geqslant\|x-y\|
$$

for $\alpha>0$. As $n \rightarrow \infty$, we have

$$
\left\|x-y-\alpha\left(x^{\prime}-y^{\prime}\right)\right\| \geqslant\|x-y\|
$$

for $\alpha>0$, which means that $A$ is dissipative in $X$. Also $\bar{A}$ is dissipative in $X$. Indeed, for $x, y \in D(\bar{A})$ and $x^{\prime} \in \bar{A} x, y^{\prime} \in \bar{A} y$, there exists $\left\{x_{n}\right\}$, $\left\{y_{n}\right\} \subset D(A)$ such that $x_{n} \rightarrow x, y_{n}^{\prime} \rightarrow y$ and $x_{n}^{\prime} \in A x_{n}, y_{n}^{\prime} \in A y_{n}$ imply $x_{n}^{\prime} \rightarrow x^{\prime}$ and $y_{n}^{\prime} \rightarrow y^{\prime}$. Since $A$ is dissipative,

$$
\left\|x_{n}-y_{n}-\alpha\left(x_{n}^{\prime}-y_{n}^{\prime}\right)\right\| \geqslant\left\|x_{n}-y_{n}\right\|
$$


for $\alpha>0$. It follows that

$$
\left\|x-y-\alpha\left(x^{\prime}-y^{\prime}\right)\right\| \geqslant\|x-y\|
$$

for $\alpha>0$ as $n \rightarrow \infty$, and from (3.10) we obtain $R(I-\alpha \bar{A}) \supset \overline{D(\bar{A})}$. By Theorem 1.3 , there exists a contraction semigroup $\{T(t) ; t \geqslant 0\}$ on $\overline{D(\bar{A})}$ such that

$$
T(t) x=\lim _{\alpha \rightarrow 0+}(I-\alpha \bar{A})^{-[t / \alpha]} x,
$$

for $x \in \overline{D(\bar{A})}$ and $\alpha>0$, and we have

$$
\|T(t) x-T(s) x\| \leqslant|t-s|\|A x\|
$$

for $x \in D(\bar{A})$. If $T(t) x$, then $x \in D(\bar{A})$ is strongly differentiable at a.e. $t \geqslant 0$, it is a unique solution of the Cauchy problem (3.11). Thus the Cauchy problem is well-posed in the sense of semigroup.

We shall show that

$$
\lim _{n \rightarrow \infty} J_{\alpha, n} x=(I-\alpha \bar{A})^{-1} x,
$$

for $x \in \overline{\operatorname{co} D(A)}$ and $\alpha>0$. For $x \in \overline{\operatorname{co} D(A)} \subset R(I-\alpha A)$, there exists $y$ $\in D(A), y^{\prime} \in A y$ such that $x=y-\alpha y^{\prime}$. From (3.8) and (3.9), for such $y$ there exists $\left\{y_{n}^{\prime}\right\}, y_{n}^{\prime} \in A_{n} y$ such that $y_{n}^{\prime} \rightarrow y^{\prime}$.

$$
\begin{aligned}
\left\|J_{\alpha, n} x-y\right\| & \leqslant\left\|J_{\alpha, n} x-J_{\alpha, n}\left(y-\alpha y_{n}^{\prime}\right)\right\| \\
& \leqslant\left\|x-y+\alpha y_{n}^{\prime}\right\| \\
& =\left\|y^{\prime}-y_{n}^{\prime}\right\| \rightarrow 0
\end{aligned}
$$

for some $\alpha>0$ as $n \rightarrow \infty$. Hence

$$
\lim _{n \rightarrow \infty} J_{\alpha, n} x=y \in D(A) \quad \text { for some } \alpha>0,
$$

that is,

$$
\lim _{n \rightarrow \infty} J_{\alpha, n} x \in D(A), x \in \overline{\operatorname{coD}(A)} \quad \text { for some } \alpha>0 .
$$

We denote the limit value of (3.15) by $J_{\alpha} x$. By Lemma 2.2, there exists 
a dissipative operator $A_{1}$ in $X$ such that $J_{\alpha}=\left(I-\alpha A_{1}\right)^{-1}$ for all $\alpha>0$. From (3.14), $\lim J_{\alpha, n}(I-\alpha A) y=y$, and $J_{\alpha}(I-\alpha A) y=y, y \in D(A)$ for $\alpha>0$ by (3.15). Since $J_{\alpha}$ is contraction, we have

$$
J_{\alpha}(I-\alpha \bar{A}) y=y, y \in D(\bar{A}) \quad \text { for } \alpha>0 .
$$

It follows from (3.16) that $D(\bar{A})=D\left(A_{1}\right)$. In fact, we obtain $x \in R\left(J_{\alpha}\right)$ $=D\left(A_{1}\right)$ for $x \in D(\bar{A})$, conversely, for $x \in D\left(A_{1}\right)=R\left(J_{\alpha}\right)$, there exists $y \in \overline{\operatorname{co} D(A)}$ such that

$$
\begin{aligned}
x & =J_{\alpha} y \\
& =J_{\alpha}(I-\alpha \bar{A})(I-\alpha \bar{A})^{-1} y \\
& =(I-\alpha \bar{A})^{-1} y \in D(\bar{A}) .
\end{aligned}
$$

Therefore from (3.16)

$$
\begin{aligned}
& (I-\alpha \bar{A}) y=\left(I-\alpha A_{1}\right) y, \\
& \bar{A} y=A_{1} y, \text { that is, } \bar{A}=A_{1} .
\end{aligned}
$$

Hence we have $J_{\alpha}=(I-\alpha \bar{A})^{-1}$ and (3.13). By the same method as in the proof of Lemma 2.3, (3.12) holds true.

REMARK 3.6. Recently H. Sunouchi [8] considered Theorem 3.4 in the case that $A, A_{n}$ are linear operators.

Department of Mathematics Pusan University, Korea

\section{References}

[1] H. BREzis and A. PAzY, Accretive sets and differential equations in Banach spaces, to appear.

[2] H. BREzis and A. PAzy, Semigroups of nonlinear contractions on convex sets, Jour. Funct. Anal. 6(1970) 237-281.

[3] M.G. CRAndall and T.M. LigGetT, Generation of semigroups of nonlinear transformations on general Banach spaces, to appear.

[4] T. KАто, Accretive operators and nonlinear evolution equations in Banach spaces, Symp. Nonlinear Functional Analysis, Chicago, Amer. Math. Soc. (1968), to appear.

[5] I. Miyadera, On the convergence of a sequence of nonlinear semigroups (in Japanese), Gakujyutsu-Kenkyu, Waseda Univ. Faculty of Education 18 (1969) 45-55. 
[6] I. Mryadera, Some remarks on semigroups on nonlinear operators, to appear.

[7] S. ÖHARU, On the generation of semigroups of nonlinear contractions, Jour. Math. Soc. Jap. 22 (1970) 526-550.

[8] H. SunouchI, Convergence of semi-discrete difference schemes of abstract Cauchy problem, Tôhoku Math. Jour. 22 (1970) 394-408.

[9] K. YoshidA, Functional Analysis, Springer Verlag (1968). 